\title{
Morfological and Structural Characteristics of II-VI Semiconductor Thin Films (ZnTe, CdTe, ZnS)
}

\author{
D. Kurbatov, ${ }^{1}$ V. Kosyak, ${ }^{1, *}$ M. Kolesnyk, ${ }^{1}$ A. Opanasyuk, ${ }^{1}$ \\ and S. Danilchenko ${ }^{2}$ \\ ${ }^{1}$ Sumy State University, Department of General and Experimental Physics, \\ Rimsky-Korsakov str. 2, UA-40007 Sumy, Ukraine \\ ${ }^{2}$ Applied Physics Instiute, Petropavlovskaya str. 58, UA-40030 Sumy, Ukraine
}

\begin{abstract}
The surface morphology and microstructural characteristic of $\mathrm{ZnTe}, \mathrm{CdTe}$ and $\mathrm{ZnS}$ thin films obtained by close-spaced sublimation technique were investigated. The structural features of layers were examined by XRD, SEM and optical microscopy. Size of coherent scattering regions, lattice microstrain and stacking fault defect concentration were estimated from X-ray diffraction line broadening. The investigation performed elucidates effect of preparation conditions on main structural characteristics of $\mathrm{ZnTe}, \mathrm{CdTe}$ and $\mathrm{ZnS}$ thin films.
\end{abstract}

Keywords: II-VI semiconductor compounds, thin films, crystal structure, lattice defects, $x$-ray diffraction (XRD), closed space sublimation (CSS)

\section{INTRODUCTION}

Thin films of II-VI compound semiconductor have drawn scientists' attention more than four decade. Such attention is related to possibility of creation number of high-performance electronic devices on basis of these thin layers [1]. But, at the same time, in most cases expectations of researchers still remain unjustified. It is largely conditioned by the structural specialty of chalcogenide thin films, which, in turn, determine electro-physical and optical properties of layers. The basic requirements, demanded for thin films are: close to stoichiometry chemical composition, single-phase and textured columnar structure 
with low concentration of stacking fault defects (SFD) and growth twins. However plenty of researches of II-VI thin films to indicate specific properties (co-existence of two polymorphic phase, zinc-blende (ZB) and wurtzite (WZ), lamellar structure of the crystallite, high concentration of SFD and growth twins, high level of microstreses and macrostreses, a tendency to anomalous axial textures formation, etc.), prevention or a removal of which is complex technological problem [2].

High-frequency cathode sputtering [3, 4], pyrolysis [4], pulse laser deposition [5] and electron-beam evaporation [6], closed space sublimation (CSS) [7-8] are only a few of technologies used for obtaining thin chalcogenide films. Due to technical features of the evaporator the CSS method allows to grow high-quality semiconductor thin films under controllable technological process. Therefore CSS method is widely used for the deposition of the II-VI compound thin films [9]. At the same time, morphology and substructure of layers obtained by CSS technique, as well as their dependence on physicaltechnological conditions of the thin film growth have not been not studied thoroughly.

\section{EXPERIMENTAL DETAILS}

Thin films were deposited on cleaned glass substrate by CSS method. The powder of the stoihiometric compounds was used as a source material. CdTe and $\mathrm{ZnTe}$ have $\mathrm{ZB}$ structure, whereas $\mathrm{ZnS}-\mathrm{WZ}$ structure with small portion of the $\mathrm{ZB}$ phase. During the thin films deposition evaporator temperature was: $\mathrm{CdTe}\left(T_{e}=893 \mathrm{~K}\right), \mathrm{ZnTe}\left(T_{e}=973 \mathrm{~K}\right)$ and $\mathrm{ZnS}\left(T_{e}=1173-1273 \mathrm{~K}\right)$. A substrate temperature was changed in a ranges: $\mathrm{CdTe}\left(T_{s}=323-823 \mathrm{~K}\right)$, $\mathrm{ZnTe}\left(T_{s}=323-773 \mathrm{~K}\right)$ and $\mathrm{ZnS}\left(T_{s}=333-973 \mathrm{~K}\right)$. The surface morphology of thin films was investigated by electron scanning microscopy and optical microscopy. The thickness of the layers $(l)$ was measured by the interference technique and by the cross-section microphotography. Jeffries method was used to determine the average grain size $(d)$, and estimation of the surface roughness was performed according to ISO/R 468 standard.

Structural studies of thin films were carried out by XRD diffractometers DRON 4-07 equipped with a Ni-filtered $K_{\alpha}$ irradiation of $\mathrm{Cu}$ anode in the range of angles $2 \theta$ from $20^{\circ}$ to $80^{\circ}$, where $2 \theta$ is Bragg angle. Focusing of X-rays was performed by Bragg-Brentano method. Phase analysis was performed by comparison of interplane distances and arbitrary intensities from the investigated samples and etalon sample according to JCPDS [10]. The texture of the layers was estimated by Harris method [11].

XRD method was also applied for determination of sizes $L$ of the coherent scattering regions (CSR) and microdeformation level $\varepsilon$ in the thin films by broadening of diffraction peaks. To distinguish the diffraction broadening caused by physical and instrumental effects the approximations of line profile 

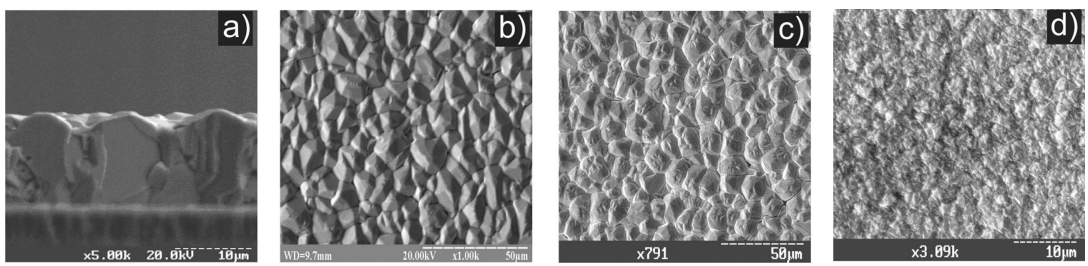

Figure 1. Microstructure of II-VI thin films: cross section of CdTe (a), surface morphology CdTe (b), ZnTe (c) and ZnS (d).

by Cauchy and Gauss were used. Calculations were carried out according to Hall method [12]. Besides that, microdeformation levels and sizes of CSR were immediately determined from the three-fold convolution describing XRD line's broadening by the method [12].

\section{RESULTS AND DISCUSSIONS}

It is determined that the structure of the films depend on the growth mechanism. The layers first grow on the substrate with a fine-grained structure with (111) oriented crystallites, parallel to the substrate (Figure 1). At low substrate temperatures low increase of crystallite size was observed and the crystallite shape of crystallite was close to equiaxial. At high substrate temperatures the mechanism of growth changed, and thin films had columnar structure (Figure 1a).

Diameter of the columnar-like grains $d$ depends on growth conditions and by the film thickness $l$. At high $T_{s}$ temperature the average grain size increases fast in the plane of layers. At same growth conditions and thickness, the $\mathrm{ZnS}$ thin films had less sizes of the crystallite than ZnTe layers. Maximal sizes of the crystallite was observed in CdTe thin films, $d \sim(0,8-1,5) l$.

Analysis of the thin films surface relief demonstrated that the surface roughness increases under increasing the substrate temperature and crystallite size. Besides at same growth conditions and thickness, $\mathrm{ZnS}$ thin films had less roughness $\left(R_{a}=0,074-0,147 \mu \mathrm{m}\right)$ than $\mathrm{ZnTe}\left(R_{a}=0,192-0,256 \mu \mathrm{m}\right)$ and CdTe $\left(R_{a}=0,296-0,364 \mu \mathrm{m}\right)$.

It is well-known that the II-VI compound layers, obtained by different techniques often have polymorphic properties i.e. have co-existence of ZB and WZ phase. That is why, we carried out advanced phase analysis of thin films.

The XRD patterns of the ZnTe, CdTe and ZnS obtained at different growth conditions are presented in Figure 2. Analysis of XRD patterns demonstrated that ZnTe layers have ZB structure, WZ phase is not observed. The same crystal structure was observed in low-temperature $\mathrm{ZnS}$ and high-temperature $\mathrm{CdTe}$ samples. As a rule, XRD patterns show reflexes from (111), (220), (311), (222), 

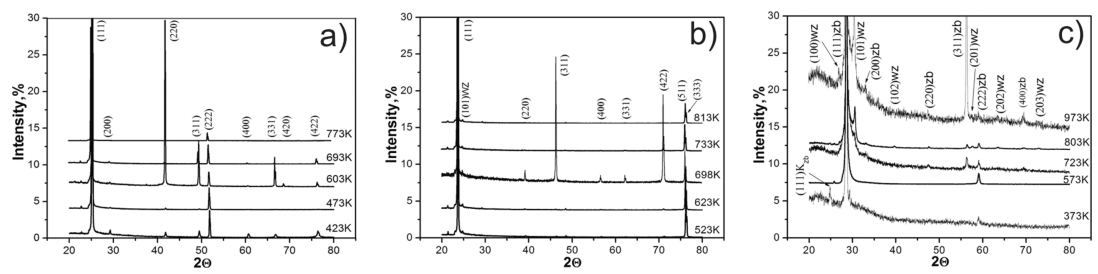

Figure 2. XRD patterns of II-VI films obtained under different condensation temperatures: ZnTe (a), CdTe (b), ZnS (c).

(331), (422) planes of ZB phase. The (111) and (222) peaks with dominant maximum intensities are present in most cases and exhibit the strong texture of the films.

At $T_{s}>573 \mathrm{~K}$ the traces of WZ phase appear in $\mathrm{ZnS}$ films, their amount slightly increases under increasing the substrate temperature (Figure 2). Peaks from WZ phase was recognized in CdTe thin films obtained at $T_{s}<500-550 \mathrm{~K}$. So high-temperature $\mathrm{ZnS}$ and low-temperature CdTe samples have two-phase structure.

Calculations by the reciprocal pole figures method allowed to determine axial growth texture [111], in chalcogenide thin films perfection of which is increased with layers thickness and depends on growth conditions. At an increase of $T_{s}$ texture perfection was raised at first, and then, at temperatures near to critical decreased to the average values.

It is known, that physical broadening of diffraction line is caused by dispersity of CSR; lattice microstrain $\varepsilon$ (as a change $\Delta d$ in the $d$-spacing in a strained sample compared to an unstrained one $\varepsilon=\Delta d / d$ ); and concentration of SFD [11-12]. The analysis of the diffraction profile with determination of the mentioned causes is well established in the investigations of polycrystalline materials but for examination of chalcogenide are used rarely. Usually diffraction line broadening is ascribed to dispersity of CSR only, which can be determined from the well-known Debye-Scherrer formula:

$$
L=\frac{k \lambda}{\beta \cos \theta}
$$

where $\lambda$ is the $\mathrm{x}$-ray wavelength, $\beta$ is the line width of the "pure" diffraction profile resulting from small crystallite size, and $k$ is a constant approximately equal to unity and related to crystallite shape.

To calculate $L$ and $\varepsilon$, the diffraction peak must be separated into components corresponding to the above mentioned sources of broadening using for example conventional approximation techniques [11]. In this research Cauchy and Gauss approximations were used to separate pure and instrumental contribution in broadening of diffraction line, as well as for simultaneous determination of $L$ and $\varepsilon$. 
The pairs of lines, (111) and (222) or (200) and (400), were chosen for estimation of microstructural parameters of ZB-phase. As revealed, the points obtained from the different pairs of lines, $(h k l)$ and $(h 00)$, belong to the different traces at Hall plot. Usually trace constructed for $(h k l)$ reflexes is higher then one constructed for $(h 00)$ reflexes. It suggest about the SFD in the (111) plates.

The values of the microstructural characteristics calculated by these approximations are in good correlation. Assuming that more precise data of $L$ and $\varepsilon$ can be obtained by the method of threefold convolution of the XRD profile, the appropriate calculation were described in [12]. The results of the calculations of $L$ and $\varepsilon$ by the method of threefold convolution for $\mathrm{ZnS}$ and CdTe are presented in Figures 3 and 4. The last data of $L$ and $\varepsilon$ are found in most cases intervening between the data obtained from Gauss and Cauchy approximations. It indicates the reliability of the results obtained from analysis of XRD line broadening.

As revealed, the calculated CSR grows in a range $\mathrm{ZnS}-\mathrm{ZnTe}-\mathrm{CdTe}$ for condensates manufactured at the identical conditions (Figure 3). At the same time $\varepsilon$ decreases in ZnTe and CdTe condensates compared to ZnS (Figure 4). For all materials at first the CSR grows as the substrate temperature $T_{s}$ increased and then revealed some lowering with further increasing of $T_{s}$. As seen, there is optimal temperature interval $\left(T_{s}=573-623 \mathrm{~K}\right)$ at which the CSR in thin films is maximal (Figure 3). The lattice microstrain in $\mathrm{ZnTe}$ and $\mathrm{ZnS}$ films decreases as $T_{s}$ is raised, and is approximately constant in CdTe films (Figure 4).

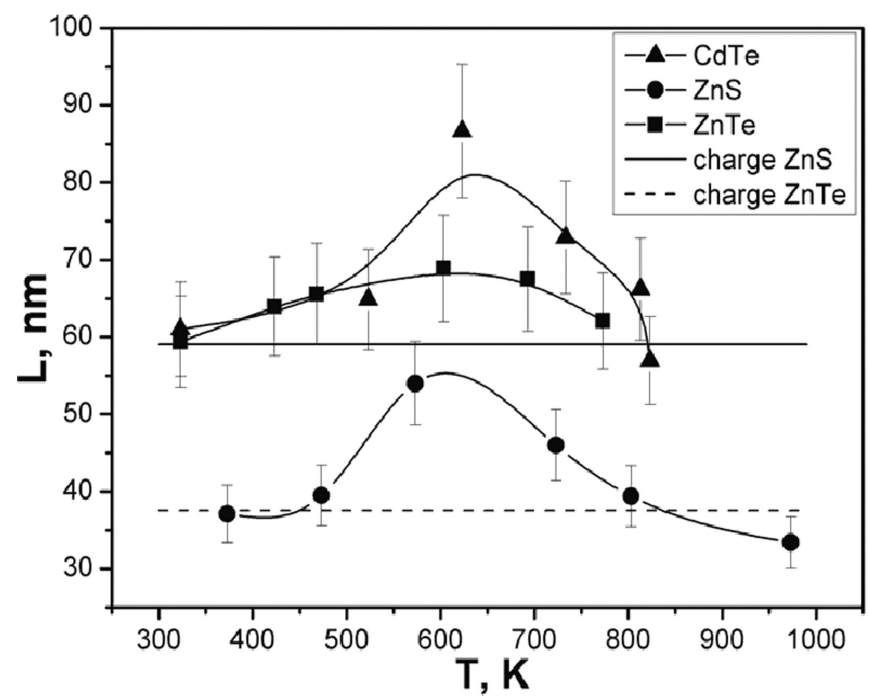

Figure 3. Effect of the substrate temperature $T_{s}$ on the scattering domain sizes $(L)$ in II-VI semiconductor compounds thin films. 


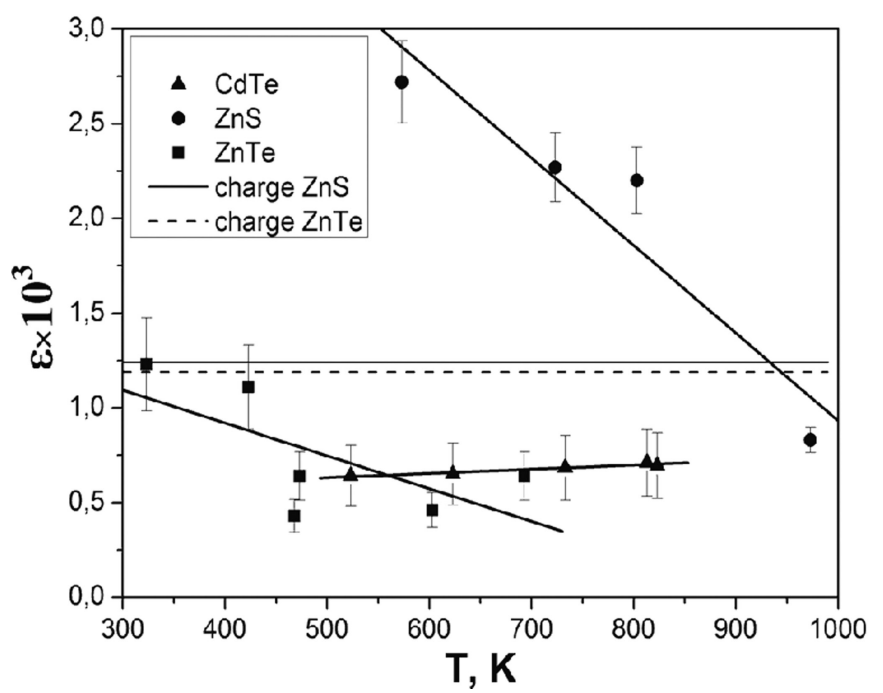

Figure 4. Effect of the substrate temperature $T_{s}$ on the microstress level $(\varepsilon)$ in II-VI semiconductor compounds thin films.

It is significant that the circumstance $L_{(h 00)}>L_{(h h h)}$ is true at all values calculated by three applied techniques. There exist two explanations of this fact: the CSR are not equidimensional and size in [200] direction is bigger then size in [111] direction; or CSR are equidimensional but addition in broadening of (111) line is caused by SFD contribution. With this assuming the calculation of the total concentration of strain SFD and growth SFD is possible by the formula:

$$
\alpha^{\prime}=1,5 \alpha_{0}+\beta_{0}=3,04\left(\frac{1}{L_{(h 00)}}-\frac{1}{L_{(h h h)}}\right) d_{(111)}
$$

$\alpha_{0}$ - concentration of strain SFD, $\beta_{0}$ - concentration of growth SFD.

Evidently, that the total concentration of SFD decreases as $T_{s}$ is raised from $0,67 \%\left(T_{s}=323 \mathrm{~K}\right)$ to $0,04 \%\left(T_{s}=693 \mathrm{~K}\right)$. The biggest concentration of SFD was found in a charge $(1,43 \%)$. Opposite tendency was observed in two-phases $\mathrm{ZnS}$ films: the concentration of SFD increases as the is $T_{s}$ raised from $0,015 \%\left(T_{s}=723 \mathrm{~K}\right)$ to $1,51 \%\left(T_{s}=973 \mathrm{~K}\right)$. The high concentration of SFD was found again in a charge of $\mathrm{ZnS}(1,22 \%)$. The data obtained are in a good agreement with XRD phase analysis, which indicate increase of WZ in $\mathrm{ZnS}$ films as $T_{s}$ is raised and excess of wurtzite in a charge of $\mathrm{ZnS}$. The calculation of SFD concentration in CdTe films was failed as a result of close values of $\mathrm{Cd}$ and $\mathrm{Te}$ atomic mass leading to blackout of (200) reflex.

As it was shown by high-resolution electron microscopy studies performed for CdTe films [13] the hexagonal phase in $\mathrm{A}_{2} \mathrm{~B}_{6}$ compounds is forming due to 
distortion of alternating order of close-packed planes, but not as separate grains: the transition from trilayered packing $A B C A B C$ typical for the zincblende structure to the bilayered ABABAB packing calls a formation of $\mathrm{WZ}$ structure. As a result the cubic matrix with a large amount of SFD and an interlaers of the hexagonal phase appears, and this matrix is conjugated $\left([110]_{\mathrm{ZB}} \|[110]_{\mathrm{WZ}}\right.$, $\left([111]_{\mathrm{ZB}} \|[001]_{\mathrm{WZ}}\right)$ with the ZB structure. This fact creates difficulties for revelation of the WZ-phase by the X-ray methods [2].

So, from our point of view, SF and specially oriented interlayers of the hexagonal phase in cubic phase of thin films defined scattering domain size along the [111] direction. Proposed model gives a good explanation of scattering domain sizes on $T_{s}$ (Figure 3) The average scattering domain sizes increases from $30-35 \mathrm{~nm}$ to $50-55 \mathrm{~nm}$ as $T_{s}$ increases from $373 \mathrm{~K}$ to $573 \mathrm{~K}$ due to growth conditions close to thermodynamically equilibrium. Besides, $L_{(111)}$ decreases owing to increasing of the SFD concentration and the amount of the wurtzite interlayers in the samples. At the same time, the crystallite size in along the [200] direction is still increased. However, unlike ZnS layers WZ phase in CdTe and $\mathrm{ZnTe}$ not observed due to their specific orientation in crystallites and low concentration.

The values of CSR are in a good correlation with [7-8, 14]. It should be noted, that scattering domain size given in $[7-8,14]$ is overestimated because authors have not separated the contributions from the different structural features. Using Debye-Scherrer law for the thin films investigation showed that scattering domain size 1,5 - 2 times higher than in the layers obtained in [7-8, 14]. At the same time maximal value of micro deformation in our thin films is two times smaller, than in $[7-8,14]$. So, results of our investigation demonstrate a high degree of structural perfection of thin films comparatively with layers obtained by other methods, including techniques similar to CSS [7-8]. Also the concentration of SFD is much smaller than in thin films obtained by other methods. It confirms availability of CSS methods for high-performance II-VI thin films deposition.

The results of investigation indicate, that structural speciality of thin films conditioned on crystal lattice thermodynamic parameters: small fluctuating of energy of the crystal lattice during change of phase ZB-WZ, low interfacial energy, as are results, to appear high probability of crystal lattice disruption. Exactly disruption lead to SFD, growth twin and WZ-phase interlayers creation. This crystal lattice defect to determine structural speciality of the thin films.

\section{SUMMARY}

The complex investigation of structural and morphological characteristics of the ZnTe, ZnS and CdTe films obtained by the CSS technology was performed by XRD, electron scanning microscopy and optical microscopy. Average scattering domain size, lattice microstrain and SFD concentration were estimated 
from XRD line broadening. The XRD-data processing was made by the conventional approximation procedure (Hall method) and approximation with threefold X-ray line convolution. The present investigation allowed determining dependencies of basic structural characteristic on the growth conditions. We defined that the structural features of thin films conditioned with the SFD. Our investigation demonstrated that the CSS technology allow to obtain II-VI compound thin films with low microstrain level and SFD concentration, big size of the crystallites and scattering domain size.

\section{REFERENCES}

1. R. Bhargava, Properties of wide band gap II-VI semiconductors. - London, United Kingdom: INSPEC, The Institution of Electrical Engineers, 1997, 238.

2. P. A. Panchekha, Structure and technology problems of $\mathrm{A}_{2} \mathrm{~B}_{6}$ semiconductor film. Functional Materials. 7(2), 1-5 (2000).

3. H. Bellakhder, A. Outzourhit, and E. L. Ameziane, Stady of ZnTe thin films deposited by r.f. sputtering. Thin Solid Films 382, 30-33 (2001).

4. B. Elidrissi, M. Addou, M. Regragui, et al. Structure, composition and optical properties of $\mathrm{ZnS}$ thin film prepared by spray pyrolysis. Mat. Chemistry and Physics 68, 175-179 (2001).

5. A. Erlacher, A. R. Lukaszew, et al. Structural and surface analysis of thinfilm ZnTe formed with pulsed-laser deposition. Surf. Sci. 600, 3762-3765 (2006).

6. S. Wang, X. Fu, G. Xia, et al. Structural and optical properties of ZnS thin films grown by glancing angle deposition. Apl. Surf. Science 252, 8734-8737 (2006).

7. S. Lalitha, R. Sathyamoorthy, S. Senthilarasu et al. Characterization of CdTe thin film-dependence of structural and optical properties on temperature and thickness. Sol. Energy Mat. Solar Cells. 82, 187-199 (2004).

8. Y. P. V. Subbaiah, P. Prathap, and K. T. Ramakrishna Reddy, Structural, electrical and optical properties of $\mathrm{ZnS}$ films deposited by close-spaced evaporation. Appl. Surf. Sci. 253, 2409-2415 (2006).

9. A. Lopez-Otero, Hot wall epitaxy. Invited Review. Thin Sol. Films 49, 3-57 (1978).

10. Selected powder diffraction data for education straining (Search manual and data cards). Published by the International Centre for diffraction data. USA. 432 (1988).

11. G. H. Stout and L. H. Jensen, X-ray structure determination: A practical guide. New York: John Wiley and Sons, p. 449 (1989).

12. S. N. Danilchenko, O. G. Kukharenko, and C. Moseke, Determination of the bone mineral crystallite size and lattice strain from diffraction line broadening. Cryst. Res. Technol. 37(11), 1234-1240 (2002). 
13. Y. Yan, M. M. Al-Jassim, K. M. Jones et al. Observation and first-principles calculation of buried wurtzite phases in zinc-blende CdTe thin films. Appl. Phys. Let. 77(10), 1461-1463 (2000).

14. T. Mahalingam, V. S. John, and G. Ravi, Microstructural Characrerization of Electrosynthesized ZnTe thin films. Cryst. Res. Technol. 37(4), 329-339 (2002). 